\title{
The Association between the Use of Proton Pump Inhibitors and the Risk of Hypomagnesemia: A Systematic Review and Meta-Analysis
}

\author{
Chan Hyuk Park', Eun Hye Kim', Yun Ho Roh², Ha Yan Kim², Sang Kil Lee ${ }^{1 *}$ \\ 1 Division of Gastroenterology, Department of Internal Medicine, Severance Hospital, Institute of Gastroenterology, Yonsei University College of Medicine, Seoul, Korea, \\ 2 Biostatistics Collaboration Unit, Yonsei University College of Medicine, Seoul, Korea
}

\begin{abstract}
Background: Although many case reports have described patients with proton pump inhibitor (PPI)-induced hypomagnesemia, the impact of PPI use on hypomagnesemia has not been fully clarified through comparative studies. We aimed to evaluate the association between the use of PPI and the risk of developing hypomagnesemia by conducting a systematic review with meta-analysis.

Methods: We conducted a systematic search of MEDLINE, EMBASE, and the Cochrane Library using the primary keywords "proton pump," "dexlansoprazole," "esomeprazole," "ilaprazole," "lansoprazole," "omeprazole," "pantoprazole," "rabeprazole," "hypomagnesemia," "hypomagnesaemia," and "magnesium." Studies were included if they evaluated the association between PPI use and hypomagnesemia and reported relative risks or odds ratios or provided data for their estimation. Pooled odds ratios with $95 \%$ confidence intervals were calculated using the random effects model. Statistical heterogeneity was assessed with Cochran's $Q$ test and $l^{2}$ statistics.

Results: Nine studies including 115,455 patients were analyzed. The median Newcastle-Ottawa quality score for the included studies was seven (range, 6-9). Among patients taking PPIs, the median proportion of patients with hypomagnesemia was $27.1 \%$ (range, $11.3-55.2 \%$ ) across all included studies. Among patients not taking PPIs, the median proportion of patients with hypomagnesemia was $18.4 \%$ (range, $4.3-52.7 \%$ ). On meta-analysis, pooled odds ratio for PPI use was found to be 1.775 (95\% confidence interval 1.077-2.924). Significant heterogeneity was identified using Cochran's $Q$ test $\left(\mathrm{df}=7, P<0.001, I^{2}=98.0 \%\right)$.
\end{abstract}

Conclusions: PPI use may increase the risk of hypomagnesemia. However, significant heterogeneity among the included studies prevented us from reaching a definitive conclusion.

Citation: Park CH, Kim EH, Roh YH, Kim HY, Lee SK (2014) The Association between the Use of Proton Pump Inhibitors and the Risk of Hypomagnesemia: A Systematic Review and Meta-Analysis. PLoS ONE 9(11): e112558. doi:10.1371/journal.pone.0112558

Editor: John Green, University Hospital Llandough, United Kingdom

Received July 24, 2014; Accepted October 8, 2014; Published November 13, 2014

Copyright: $\odot 2014$ Park et al. This is an open-access article distributed under the terms of the Creative Commons Attribution License, which permits unrestricted use, distribution, and reproduction in any medium, provided the original author and source are credited.

Data Availability: The authors confirm that all data underlying the findings are fully available without restriction. All relevant data are within the paper.

Funding: The authors have no funding or support to report.

Competing Interests: The authors have declared that no competing interests exist.

* Email: sklee@yuhs.ac

\section{Introduction}

Proton pump inhibitors (PPIs) are a mainstay of treatment for acid-related diseases, including gastroesophageal reflux disease, functional dyspepsia, and peptic ulcer disease [1-5]. PPIs have an excellent safety profile, and have become one of the most commonly prescribed class of drugs in both primary and specialty care [6]. However, PPI use may induce some adverse events, including interstitial nephritis [7], respiratory infections [8], Clostridium difficile colitis [9], and hip fractures [10]. More recently, it has been reported that hypomagnesemia may also be induced by PPIs. The association between symptomatic hypomagnesemia and PPI use was first described in two patients in 2006 [11]. Since this report, many case reports have accumulated, supporting the association between PPI use and induced hypomagnesemia [12-16]. The Food and Drug Administration issued a Drug Safety Communication in 2011 [17], emphasizing the importance of long term use of prescription PPIs in this association. Moreover, a systematic review of 18 case reports of PPI-induced hypomagnesemia found that discontinuation of PPI use resulted in recovery from PPI-induced hypomagnesemia [18]. Although the pathophysiology of PPI-induced hypomagnesemia has not been definitively determined, impaired absorption of intestinal magnesium, due to administration of PPIs, may be one possible mechanism [19]. Decreased luminal $\mathrm{pH}$ in the intestine, caused by PPI use, may alter the affinity of the transient receptor potential melastatin- 6 and transient receptor potential melastatin7 (TRPM6/7) channel for $\mathrm{Mg}^{2+}$, reducing active transport of $\mathrm{Mg}^{2+}[20,21]$.

Although many case reports have described patients with PPIinduced hypomagnesemia, the impact of PPI use on hypomagnesemia has not been fully clarified in comparative studies [22-30]. 


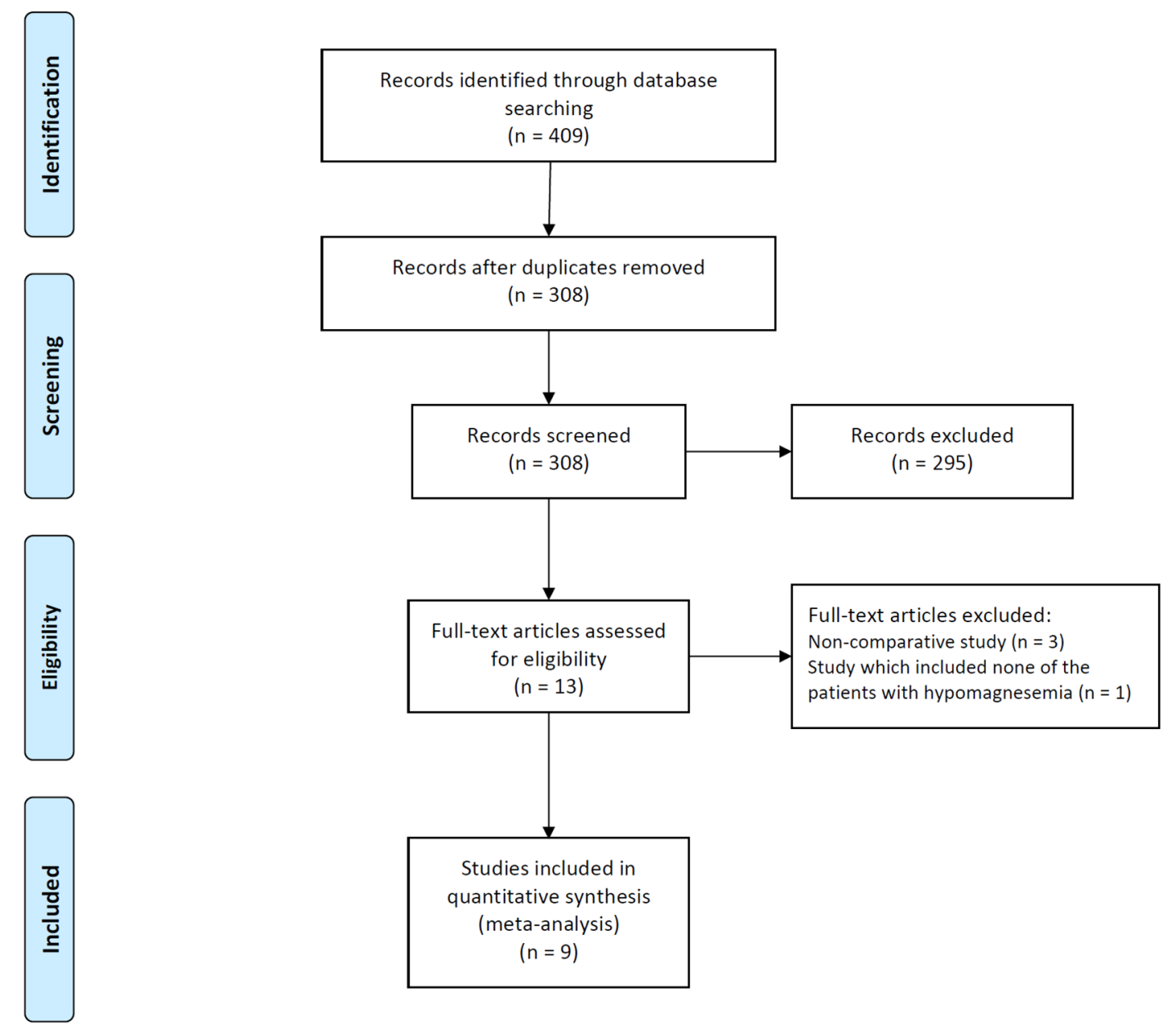

Figure 1. Flow diagram of studies included in the meta-analysis. PPI, proton pump inhibitor. doi:10.1371/journal.pone.0112558.g001

In some studies, the association between PPI use and hypomagnesemia was not identified [23-25,29], while in others, PPI use was found to increase the risk of hypomagnesemia $[22,26-28,30]$. The discrepancy between these studies may be attributed to various patient characteristics, and/or underlying conditions.

To examine this topic, we performed a systematic review with meta-analysis, of existing comparative studies that investigated the association between PPI use and the risk of developing hypomagnesemia.

\section{Materials and Methods}

\section{Search strategy}

We searched for all relevant studies published between January 1990 and May 2014 that examined the effect of PPI use on the risk of hypomagnesemia, using MEDLINE, EMBASE, and the Cochrane Library. The terms "proton pump," "dexlansoprazole," "esomeprazole," "ilaprazole," "lansoprazole," "omeprazole," "pantoprazole," "rabeprazole," "hypomagnesemia," "hypomagnesaemia," and "magnesium" were used in our search. We also examined the references of screened articles, in order to identify additional studies. All human studies published in English were considered, and the latest date for updating our search was August 19, 2014.

\section{Study selection}

In the first stage of the study selection, the titles and abstracts of papers returned by our keyword search were examined to exclude irrelevant articles. Next, the full-text of all selected studies was screened, according to our inclusion and exclusion criteria. The inclusion criteria specified (1) studies regarding PPIs and hypomagnesemia, and (2) studies reporting relative risks or odds ratios (ORs) of PPI use, or provided data for their calculation. The exclusion criteria ruled out publications in a language other than English. Two investigators (C.H.P. and E.H.K.) independently evaluated studies for eligibility, resolved any disagreements through discussion and consensus. If no agreement could be reached, a third investigator (S.K.L.) decided eligibility.

To understand the risk of bias in individual studies, a formal quality assessment of studies was performed, along with subgroup analysis. The methodological quality of observational studies was independently assessed by two investigators (C.H.P. and E.H.K.), using the Newcastle-Ottawa scale [31,32]. Using this scale, observational studies were scored across three categories: selection (4 questions), comparability of study groups (2 questions), and ascertainment of the exposure or outcome (3 questions). All questions were assigned a score of one point, with the exception of comparability of study groups, in which a maximum of two points were awarded. Studies with a cumulative score $\geq 7$ were considered high quality [33,34]. 


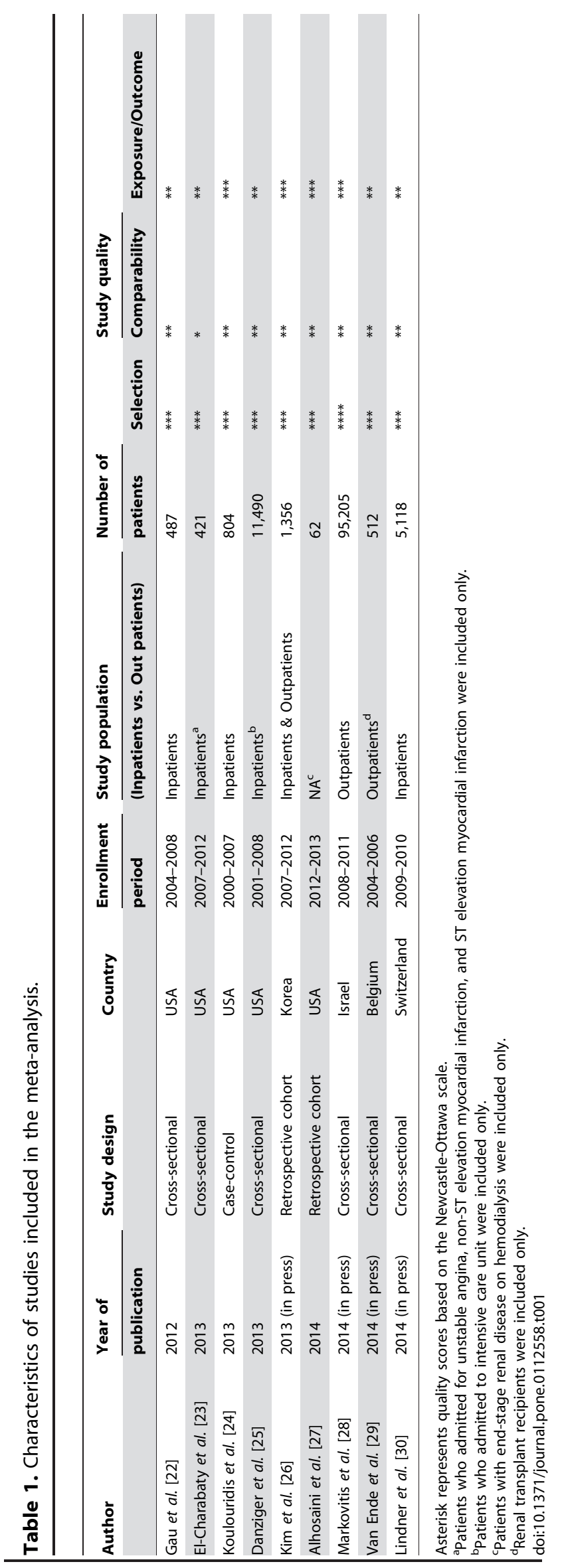

\section{Data extraction}

Using a data extraction form developed in advance, two reviewers (C.H.P. and E.H.K.) independently extracted the following information: first author, year of publication, study design, country, enrollment period, study population, age and sex of patients, definition of hypomagnesemia, total number of patients in each group (exposed vs. not exposed), ORs, and 95\% confidence intervals (CIs). When incomplete information was available, attempts were made to contact the corresponding authors of the studies for additional information.

\section{Outcomes assessed}

Our primary analysis focused on assessing the risk of hypomagnesemia among users of PPIs. Our a priori hypothesis included study population (inpatients vs. others) as a potential explanation for heterogeneity in the direction and magnitude of effect. For exploratory analysis we conducted subgroup analysis according to the cut-off value of serum magnesium level $(1.7 \mathrm{mg}$ / $\mathrm{dL}$ vs. $1.8 \mathrm{mg} / \mathrm{dL}$ ).

\section{Statistical analysis}

Meta-analyses were performed to calculate pooled ORs with 95\% CIs [35]. Taking a conservative approach, we used a random effects model, which produces wider CIs than a fixed effect model. We assessed heterogeneity using two methods: Cochran's Q test, which was considered statistically significant for heterogeneity if $P<0.1$, and $I^{2}$ statistics, with values $>50 \%$ suggestive of significant heterogeneity [36]. The tests for funnel plot asymmetry was not conducted when the included studies were less than 10 [37]. All $P$ values were 2-tailed, and a value of $P<0.05$ was considered statistically significant for all tests (except heterogeneity). Analysis and reporting were performed according to the Preferred Reporting Items for Systematic Reviews and Meta-Analyses (PRISMA) guidelines [38]. Statistical analyses were conducted using the statistical software Comprehensive Meta Analysis (version 2.2.064; Biostat Inc., Englewood, NJ, USA).

\section{Results}

\section{Study selection}

A flow diagram for our systematic review is shown in Fig. 1. In summary, 409 studies were identified by our literature search. After scanning titles and abstracts, we discarded 101 duplicate articles retrieved through multiple search engines. Another 295 irrelevant articles were excluded based on the titles and abstracts. The full texts of the 13 remaining articles were reviewed, and 4 non-pertinent articles were excluded. Of 4 excluded studies, three were non-comparative studies without control group and remaining one included none of the patients with hypomagnesemia in both treatment and control group.

Of the original 409 studies, nine studies were deemed appropriate for our meta-analysis [22-25,27,28]. These studies included a total of 115,455 patients, and all had been published in the past 2 years, between 2012 and 2014. The enrollment period for these studies ranged from 2000 to 2013, and all were retrospective in nature. Of the included studies, five included only hospitalized patients [22-25,30], two included outpatients only $[28,29]$, one included both inpatients and outpatients [26], and one included patients with end-stage renal disease on hemodialysis, but did not provide data regarding the hospitalization of patients [27]. The median Newcastle-Ottawa quality score for these studies was seven (range, 6-9), and all studies except one were considered high quality. The characteristics of the studies included in the meta-analysis are summarized in Table 1. 


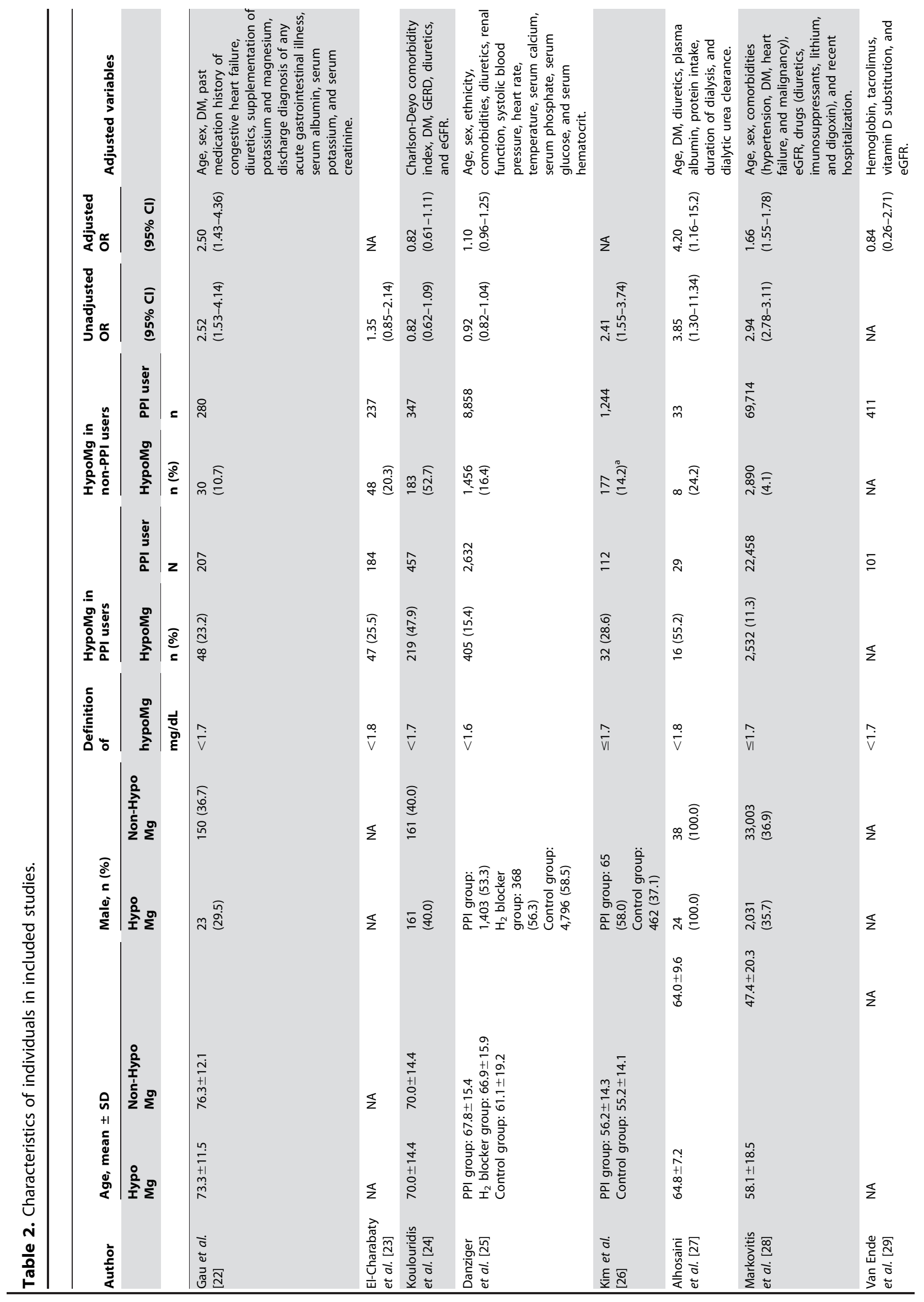




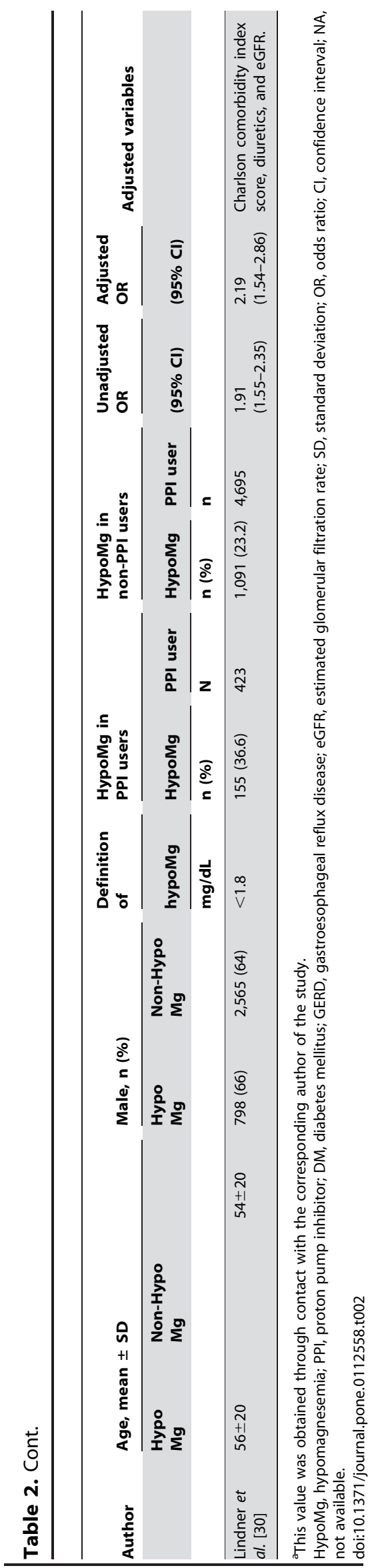

\section{Risk of hypomagnesemia}

The cut-off value of serum magnesium level for defining hypomagnesemia was $1.6,1.7$, and $1.8 \mathrm{mg} / \mathrm{dL}$ in one [25], five $[22,24,26,28,29]$, and three studies $[23,27,30]$, respectively (Table 2). Among patients taking PPIs, the median proportion of patients with hypomagnesemia in all included studies was $27.1 \%$ (range, 11.3-55.2\%). In addition, the median of the proportion of patients with hypomagnesemia in those not taking PPIs was $18.4 \%$ (range, 4.3-52.7\%) across the studies. Of 9 included studies, six reported both unadjusted OR and adjusted OR. Other two studies showed unadjusted OR only. Remaining one study showed adjusted OR only. Most studies adjusted for the following confounders: use of diuretics (6/7), renal function (5/7), age $(4 / 7)$, diabetes mellitus (4/7), and comorbidities (4/7). On metaanalysis, pooled unadjusted OR for PPI use was found to be 1.775 (95\% CI $=1.077-2.924)$. Significant heterogeneity was identified (Cochran's Q test, $\mathrm{df}=7, P<0.001, I^{2}=98.0 \%$ ). This risk increase with PPI use persisted even after adjusting for potential confounders where reported in studies (pooled adjusted OR $[95 \% \mathrm{CI}]=1.484[1.103-1.997]$, Fig. 2), although the heterogeneity persisted (Cochran's Q test, df $=6, P<0.001, I^{2}=89.1 \%$ ).

\section{Subgroup analysis}

We performed pre-planned subgroup analysis of studies, based on the hospitalization of patients. In five studies which included only hospitalized patients [22-25,30], PPI use was not associated with hypomagnesemia (pooled unadjusted OR [95\% CI] $=1.342$ [0.895-2.011]). Significant heterogeneity did exist in the inpatient subgroup (Cochran's Q test, df $=4, P<0.001, I^{2}=92.0 \%$ ). Pooled adjusted $\mathrm{OR}$ showed similar results between PPI use and incidence of hypomagnesemia compared to pooled unadjusted OR (pooled adjusted OR [95\% CI] $=1.424$ [0.924-2.196], Fig. 3). Significant heterogeneity persisted in this analysis (Cochran's Q test, $\mathrm{df}=3$, $\left.P<0.001, I^{2}=88.3 \%\right)$.

For exploratory analysis, we performed additional subgroup analysis according to the cut-off value of serum magnesium level. In 4 studies which reported adjusted OR based on the $1.7 \mathrm{mg} / \mathrm{dL}$ of cut-off value [22,24,28,29], PPI use was not associated with incidence of hypomagnesemia (pooled adjusted OR [95\% $\mathrm{CI}]=1.363$ [0.827-2.246], Fig. 4A). Significant heterogeneity was identified in this subgroup (Cochran's $Q$ test, $\mathrm{df}=3, P<$ $0.001, I^{2}=87.7 \%$ ). In 2 studies which showed adjusted ORs based on the $1.8 \mathrm{mg} / \mathrm{dL}$ of cut-off value $[27,30]$, on the contrary, PPI use increased the risk of hypomagnesemia (pooled adjusted OR $[95 \% \mathrm{CI}]=2.292[1.632-3.218]$, Fig. 4B). There was no heterogeneity in this subgroup (Cochran's $Q$ test, $\mathrm{df}=1, P=0.339$, $\left.I^{2}=0.0 \%\right)$.

\section{Discussion}

Intracellular magnesium is an important cofactor for enzymatic reactions, and is critical for energy metabolism involving adenosine triphosphate [19]. Magnesium homeostasis is determined primarily by two processes, gastrointestinal absorption and renal excretion [39]. Gastrointestinal magnesium absorption occurs through both passive paracellular movement, and active transport into the portal venous system [19]. Active magnesium transport in the gut occurs through the combined action of TRPM6/7 channels, which are present in the apical membrane of enterocytes [39]. Previous studies have found that renal excretion of magnesium is reduced appropriately in patients with PPIinduced hypomagnesemia [40,41], and that a PPI-induced decrease in the luminal $\mathrm{pH}$ of the intestine might alter the TRPM6/7 channel's affinity for magnesium [21]. Therefore, 


\begin{tabular}{lcccrrr} 
Study name & \multicolumn{5}{c}{ Statistics for each study } \\
& $\begin{array}{l}\text { Odds } \\
\text { ratio }\end{array}$ & $\begin{array}{c}\text { Lower } \\
\text { limit }\end{array}$ & $\begin{array}{c}\text { Upper } \\
\text { limit }\end{array}$ & Z-Value & p-Value \\
Gau [22] & 2.500 & 1.430 & 4.371 & 3.215 & 0.001 \\
Koulouridis [24] & 0.820 & 0.610 & 1.102 & -1.315 & 0.189 \\
Danziger [25] & 1.100 & 0.960 & 1.260 & 1.372 & 0.170 \\
Alhosaini [27] & 4.200 & 1.160 & 15.207 & 2.186 & 0.029 \\
Markovitis [28] & 1.660 & 1.550 & 1.778 & 14.488 & 0.000 \\
Van Ende [29] & 0.840 & 0.260 & 2.714 & -0.291 & 0.771 \\
Lindner [30] & 2.190 & 1.540 & 3.114 & 4.363 & 0.000 \\
& 1.484 & 1.103 & 1.997 & 2.608 & 0.009
\end{tabular}

Figure 2. Forest plots for risk of hypomagnesemia. PPI, proton pump inhibitor; $\mathrm{Cl}$, confidence interval. doi:10.1371/journal.pone.0112558.g002

impaired intestinal absorption rather than renal excretion is considered the primary cause of PPI-induced hypomagnesemia.

Many previous case reports [12-16], and proposed mechanisms of PPI-induced hypomagnesemia, have promoted awareness of the risk of hypomagnesemia in patients taking PPIs. However, the risk for PPI-induced hypomagnesemia should be evaluated by comparative studies, since the prevalence of hypomagnesemia is not rare. One previous population-based study found that $2 \%$ of subjects (104 of 5,179) have hypomagnesemia [42]. Other population-based studies also have suggested that hypomagnesemia is not rare in the general population $[43,44]$. Moreover, in hospitalized patients, electrolyte disorders (including hypomagnesemia) are often acute and severe. Therefore, case reports alone cannot analyze the effect of PPI use on hypomagnesemia, and well-designed, comparative studies are needed to clarify the risk of PPI-induced hypomagnesemia.

Our meta-analysis showed statistical significance between PPI use and the risk of hypomagnesemia. The risk of hypomagnesemia in PPI users persisted even after adjusting for confounding variables. However, the significant heterogeneity among the included studies may be a concern. The significance of heterogeneity implied that the effect of PPI use on hypomagnesemia was varied. The observed heterogeneity may be due to the various types of study design and population among our included studies. For example, Gau et al.'s study [22], Koulouridis et al.'s study [24], and Lindner et al.'s study [30] included hospitalized patients, regardless of disease type. However, Koulouridis et al.'s study was designed as case-control study, while other two studies were designed as cross-sectional study. In contrast, Danziger et al.'s study [25] included only patients admitted to intensive care units. El-Charabaty et al.'s study [23] included only hospitalized patients with unstable angina, non-ST elevation myocardial infarction, and
ST elevation myocardial infarction. Kim et al.'s study [26] included both inpatients and outpatients. Alhosaini et al.'s study [27] included only patients with end-stage renal disease on hemodialysis. Van Ende et al.'s study [29] included only renal transplant recipients. Finally, Markovitis et al.'s study [28] included only outpatients in the community setting. In addition, the definition of hypomagnesemia was varied among the included studies. These variations in study design may be reflected in the proportion of patients with hypomagnesemia for each group (patients taking PPIs vs. those not taking PPIs). The proportion of patients with hypomagnesemia ranged from $11.3 \%$ to $55.2 \%$ (PPI user group), and from $4.1 \%$ to $52.7 \%$ (non-PPI user group), and therefore, we believe that the incidence or prevalence of hypomagnesemia may depend on the study design.

One of the clinical concerns on PPI-induced hypomagnesemia is whether the test for serum magnesium level should be performed before initiating PPIs or not. Although it has not been fully evaluated, experts usually recommend the test for serum magnesium level prior to initiation of PPIs when patients are expected to be on treatment for long period of time $[45,46]$. While short-term standard dose PPI treatment has low risk, long-term PPI use may complicate health conditions in high risk patients for hypomagnesemia including elderly patients [46]. The inter-study differences in the proportion of patients with hypomagnesemia in our meta-analysis may suggest that there are patients with increased susceptibility to PPI-induced hypomagnesemia. Considering the excellent safety profile of PPIs, risk identification and stratification for PPI-induced hypomagnesemia may be more helpful for clinical practice, rather than investigation of PPIinduced hypomagnesemia in the general population. Kim et al.'s study, which evaluated associated factors for hypomagnesemia, found that long-term PPI use ( $>1$ year), young age $(<45$ years),

\begin{tabular}{lcccrrr} 
Study name & \multicolumn{5}{c}{ Statistics for each study } \\
& $\begin{array}{l}\text { Odds } \\
\text { ratio }\end{array}$ & $\begin{array}{c}\text { Lower } \\
\text { limit }\end{array}$ & $\begin{array}{c}\text { Upper } \\
\text { limit }\end{array}$ & Z-Value & p-Value \\
Gau [22] & 2.500 & 1.430 & 4.371 & 3.215 & 0.001 \\
Koulouridis [24] & 0.820 & 0.610 & 1.102 & -1.315 & 0.189 \\
Danziger [25] & 1.100 & 0.960 & 1.260 & 1.372 & 0.170 \\
Lindner [30] & 2.190 & 1.540 & 3.114 & 4.363 & 0.000 \\
& 1.424 & 0.924 & 2.196 & 1.601 & 0.109
\end{tabular}

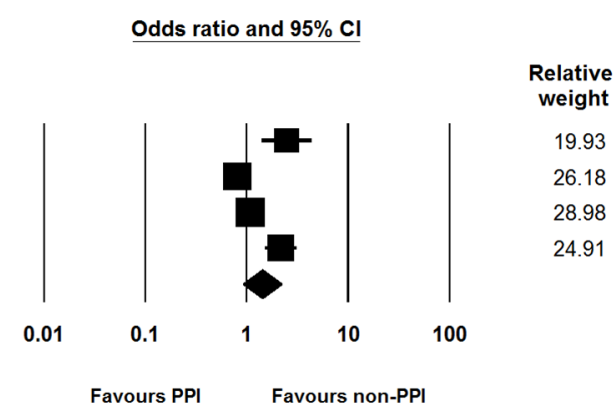

Figure 3. Subgroup analysis for studies which included only hospitalized patients. PPI, proton pump inhibitor; $\mathrm{Cl}$, confidence interval. doi:10.1371/journal.pone.0112558.g003 
A Study name

\begin{tabular}{lcccccc}
\cline { 1 - 5 } Study name & \multicolumn{5}{c}{ Statistics for each study } \\
& $\begin{array}{l}\text { Odds } \\
\text { ratio }\end{array}$ & $\begin{array}{c}\text { Lower } \\
\text { limit }\end{array}$ & $\begin{array}{c}\text { Upper } \\
\text { limit }\end{array}$ & z-Value & p-Value \\
Gau [22] & 2.500 & 1.430 & 4.371 & 3.215 & 0.001 \\
Koulouridis [24] & 0.820 & 0.610 & 1.102 & -1.315 & 0.1 \\
Markovitis [28] & 1.660 & 1.550 & 1.778 & 14.488 & 0.000 \\
Van Ende [29] & 0.840 & 0.260 & 2.714 & -0.291 & 0.771 \\
& 1.363 & 0.827 & 2.246 & 1.213 & 0.225
\end{tabular}

\begin{tabular}{lccccc} 
Study name & \multicolumn{5}{c}{ Statistics for each study } \\
& $\begin{array}{l}\text { Odds } \\
\text { ratio }\end{array}$ & $\begin{array}{c}\text { Lower } \\
\text { limit }\end{array}$ & $\begin{array}{c}\text { Upper } \\
\text { limit }\end{array}$ & Z-Value & p-Value \\
Alhosaini [27] & 4.200 & 1.160 & 15.207 & 2.186 & 0.029 \\
Lindner [30] & 2.190 & 1.540 & 3.114 & 4.363 & 0.000 \\
& 2.292 & 1.632 & 3.218 & 4.786 & 0.000
\end{tabular}

\section{alue 0.001 0.189 0.000 0.771 0.225}

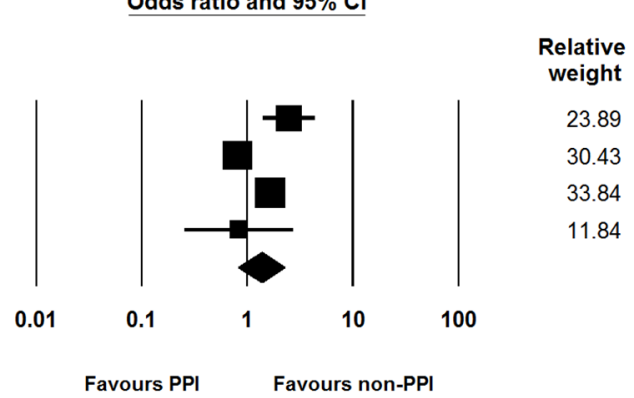

Odds ratio and $95 \% \mathrm{Cl}$

Odds ratio and $95 \% \mathrm{CI}$

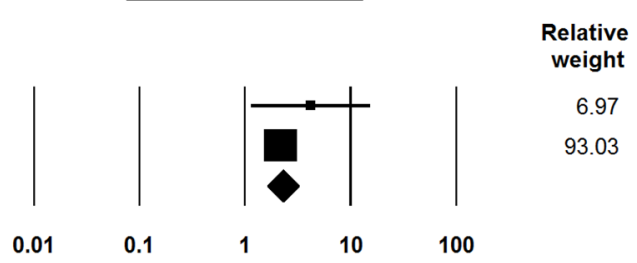

Favours PPI Favours non-PPI

Figure 4. Subgroup analysis according to the cut-off value of serum magnesium levels. (A) Subgroup analysis for studies based on the $1.7 \mathrm{mg} / \mathrm{dL}$ of cut-off value. (B) Subgroup analysis for studies based on the $1.8 \mathrm{mg} / \mathrm{dL}$ of cut-off value. PPI, proton pump inhibitor; $\mathrm{Cl}$, confidence interval.

doi:10.1371/journal.pone.0112558.g004

and concurrent cisplatin or carboplatin use were associated with hypomagnesemia in PPI users [26]. However, the study only included PPI users whose serum magnesium levels were available, and therefore, selection bias may be a concern. A population based study with regular checkup for serum magnesium level may be needed for clarifying the high risk patients for PPI-induced hypomagnesemia.

In our study, we conducted the two types of subgroup analysis. The former was subgroup analysis for studies which included hospitalized patients only, while the latter was subgroup analysis according to the cut-off value of serum magnesium level. In the former subgroup analysis, statistical significance between PPI use and the risk of hypomagnesemia was not shown. Although the subgroup included only hospitalized patients, the variation among the studies was still existed. In the latter subgroup analysis, PPI use increased the risk of hypomagnesemia in the studies whose cut-off value was $1.8 \mathrm{mg} / \mathrm{dL}$ rather than $1.7 \mathrm{mg} / \mathrm{dL}$. These results implied that PPI-induced hypomagnesemia might not be as severe as we were concerned; however, further studies would be needed for assessing the severity of PPI-induced hypomagnesemia.

Although this is the first meta-analysis which demonstrated that PPI use could increase the risk of hypomagnesemia, there are several limitations. First, this is the meta-analysis for observational studies rather than randomized controlled trials. Magnesium assessment in a large database is usually healthcare-driven and potentially biased. In addition, dyspepsia may lead to prescribing PPIs as well as deficient food intake with low magnesium ingestion. Furthermore, serum magnesium was not usually evaluated in clinical practice. Although we conducted a meta-analysis using the adjusted ORs in the included studies, potential issues of

\section{References}

1. Lacy BE, Talley NJ, Locke GR, Bouras EP, DiBaise JK, et al. (2012) Review article: current treatment options and management of functional dyspepsia. Alimentary pharmacology \& therapeutics 36: 3-15. confounding variables may be exist. The conclusion from the meta-analysis for observational studies should be interpreted carefully. Second, the significant heterogeneity among the included studies was additional obvious limitation. Through our systematic review and meta-analysis, we found that the proportion of patients with hypomagnesemia depended on the study settings including patient population and characteristics. Prospective cohort studies will be needed to evaluate severity of PPI-induced hypomagnesemia and to identify high risk group for PPI-induced hypomagnesemia. Third, we could not assess the risk of PPIinduced hypomagnesemia according to the amount or duration of usage of PPIs because available data were limited in the included studies.

Despite of these limitations, our meta-analysis showed that PPI use may increase the risk of hypomagnesemia. However, significant heterogeneity among the included studies prevented us from reaching a definitive conclusion. Well-designed, prospective cohort studies, which include regular serum magnesium monitoring, would provide more conclusive results.

\section{Supporting Information}

\section{Ghecklist S1 PRISMA checklist of the study.} (DOC)

\section{Author Contributions}

Conceived and designed the experiments: CHP SKL. Performed the experiments: CHP EHK SKL. Analyzed the data: CHP YHR HYK. Contributed reagents/materials/analysis tools: CHP. Contributed to the writing of the manuscript: CHP.
2. Yeomans ND, Tulassay Z, Juhasz L, RÃcz I, Howard JM, et al. (1998) A comparison of omeprazole with ranitidine for ulcers associated with nonsteroidal antiinflammatory drugs. Acid Suppression Trial: Ranitidine versus Omeprazole 
for NSAID-associated Ulcer Treatment (ASTRONAUT) Study Group. The New England journal of medicine 338: 719-726.

3. Hawkey CJ, Karrasch JA, Szczepanski L, Walker DG, Barkun A, et al. (1998) Omeprazole compared with misoprostol for ulcers associated with nonsteroidal antiinflammatory drugs. Omeprazole versus Misoprostol for NSAID-induced Ulcer Management (OMNIUM) Study Group. The New England journal of medicine 338: 727-734.

4. Kahrilas PJ, Shaheen NJ, Vaezi MF, Hiltz SW, Black E, et al. (2008) American Gastroenterological Association Medical Position Statement on the management of gastroesophageal reflux disease. Gastroenterology 135: 1383-1391, 1391. e1381.

5. Xiao Y, Peng S, Tao J, Wang A, Lin J, et al. (2010) Prevalence and symptom pattern of pathologic esophageal acid reflux in patients with functional dyspepsia based on the Rome III criteria. The American journal of gastroenterology 105: 2626-2631.

6. Sheen E, Triadafilopoulos G (2011) Adverse effects of long-term proton pump inhibitor therapy. Digestive diseases and sciences 56: 931-950.

7. Sierra F, Suarez M, Rey M, Vela MF (2007) Systematic review: Proton pump inhibitor-associated acute interstitial nephritis. Alimentary pharmacology \& therapeutics 26: 545-553.

8. Herzig SJ, Howell MD, Ngo LH, Marcantonio ER (2009) Acid-suppressive medication use and the risk for hospital-acquired pneumonia. JAMA: the Journal of the American Medical Association 301: 2120-2128.

9. Howell MD, Novack V, Grgurich P, Soulliard D, Novack L, et al. (2010) Iatrogenic gastric acid suppression and the risk of nosocomial Clostridium difficile infection. Archives of internal medicine 170: 784-790.

10. Gray SL, LaCroix AZ, Larson J, Robbins J, Cauley JA, et al. (2010) Proton pump inhibitor use, hip fracture, and change in bone mineral density in postmenopausal women: results from the Women's Health Initiative. Archives of internal medicine 170: 765-771.

11. Epstein M, McGrath S, Law F (2006) Proton-pump inhibitors and hypomagnesemic hypoparathyroidism. N Engl J Med 355: 1834-1836.

12. Cundy T, Dissanayake A (2008) Severe hypomagnesaemia in long-term users of proton-pump inhibitors. Clinical endocrinology 69: 338-341.

13. Broeren MA, Geerdink EA, Vader HL, van den Wall Bake AW (2009) Hypomagnesemia induced by several proton-pump inhibitors. Annals of Internal Medicine 151: 755-756.

14. Kuipers MT, Thang HD, Arntzenius AB (2009) Hypomagnesaemia due to use of proton pump inhibitors-a review. Netherlands journal of medicine 67: 169172.

15. Hoorn EJ, van der Hoek J, de Man RA, Kuipers EJ, Bolwerk C, et al. (2010) A case series of proton pump inhibitor-induced hypomagnesemia. American journal of kidney diseases 56: 112-116.

16. Regolisti G, Cabassi A, Parenti E, Maggiore U, Fiaccadori E (2010) Severe hypomagnesemia during long-term treatment with a proton pump inhibitor. American journal of kidney diseases 56: 168-174.

17. FDA Drug Safety Communication: Low magnesium levels can be associated with long-term use of Proton Pump Inhibitor drugs. Available at: http://www. fda.gov/Drugs/DrugSafety/ucm245011. Accessed May 25, 2014.

18. Hess MW, Hoenderop JG, Bindels RJ, Drenth JP (2012) Systematic review: hypomagnesaemia induced by proton pump inhibition. Alimentary pharmacology \& therapeutics 36: 405-413.

19. Perazella MA (2013) Proton pump inhibitors and hypomagnesemia: a rare but serious complication. Kidney international 83: 553-556.

20. Michalek W, Semler JR, Kuo B (2011) Impact of acid suppression on upper gastrointestinal $\mathrm{pH}$ and motility. Digestive diseases and sciences 56: 1735-1742.

21. Bai JP, Hausman E, Lionberger R, Zhang X (2012) Modeling and simulation of the effect of proton pump inhibitors on magnesium homeostasis. 1. Oral absorption of magnesium. Mol Pharm 9: 3495-3505.

22. Gau JT, Yang YX, Chen R, Kao TC (2012) Uses of proton pump inhibitors and hypomagnesemia. Pharmacoepidemiol Drug Saf 21: 553-559.

23. El-Charabaty E, Saifan C, Abdallah M, Naboush A, Glass D, et al. (2013) Effects of proton pump inhibitors and electrolyte disturbances on arrhythmias. Int J Gen Med 6: 515-518.

24. Koulouridis I, Alfayez M, Tighiouart H, Madias NE, Kent DM, et al. (2013) Out-of-hospital use of proton pump inhibitors and hypomagnesemia at hospital admission: a nested case-control study. American journal of kidney diseases 62 : $730-737$.
25. Danziger J, William JH, Scott DJ, Lee J, Lehman L, et al. (2013) Proton-pump inhibitor use is associated with low serum magnesium concentrations. Kidney international 83: 692-699.

26. Kim S, Lee H, Park CH, Shim CN, Lee HJ, et al. (2013) Clinical Predictors Associated With Proton Pump Inhibitor-Induced Hypomagnesemia. Am J Ther.

27. Alhosaini M, Walter JS, Singh S, Dieter RS, Hsieh A, et al. (2014) Hypomagnesemia in hemodialysis patients: role of proton pump inhibitors. American journal of nephrology 39: 204-209.

28. Markovits N, Loebstein R, Halkin H, Bialik M, Landes-Westerman J, et al. (2014) The association of proton pump inhibitors and hypomagnesemia in the community setting. J Clin Pharmacol.

29. Van Ende C, Van Laecke S, Marechal C, Verbeke F, Kanaan N, et al. (2014) Proton-pump inhibitors do not influence serum magnesium levels in renal transplant recipients. J Nephrol.

30. Lindner G, Funk GC, Leichtle AB, Fiedler GM, Schwarz C, et al. (2014) Impact of proton pump inhibitor use on magnesium homoeostasis: a cross-sectional study in a tertiary emergency department. Int J Clin Pract.

31. Wells GA, Shea B, O'Connell D, Peterson J, Welch V, et al. The NewcastleOttawa Scale (NOS) for assessing the quality of nonrandomised studies in metaanalyses. Available at: http://www.ohri.ca/programs/clinical_epidemiology/ oxford.asp. Accessed May 25, 2014

32. Stang A (2010) Critical evaluation of the Newcastle-Ottawa scale for the assessment of the quality of nonrandomized studies in meta-analyses. Eur J Epidemiol 25: 603-605.

33. Castillo J, Mull N, Reagan JL, Nemr S, Mitri J (2012) Increased incidence of non-Hodgkin lymphoma, leukemia, and myeloma in patients with diabetes mellitus type 2: a meta-analysis of observational studies. Blood 119: 4845-4850.

34. Singh S, Singh PP, Singh AG, Murad MH, Sanchez W (2013) Statins are associated with a reduced risk of hepatocellular cancer: a systematic review and meta-analysis. Gastroenterology 144: 323-332.

35. DerSimonian R, Laird N (1986) Meta-analysis in clinical trials. Controlled clinical trials 7: 177-188.

36. Higgins JP, Thompson SG, Deeks J, Altman DG (2003) Measuring inconsistency in meta-analyses. BMJ. British medical journal 327: 557-560.

37. Higgins J, Green S (2011) Cochrane handbook for systematic reviews of interventions, Version 5.1.0, Available at: http://handbook.cochrane.org/ chapter_10/10_4_3_1_recommendations_on_testing_for_funnel_plot_ asymmetry.htm. Accessed August 29, 2014.

38. Liberati A, Altman DG, Tetzlaff J, Mulrow C, GÃtzsche PC, et al. (2009) The PRISMA statement for reporting systematic reviews and meta-analyses of studies that evaluate health care interventions: explanation and elaboration. Journal of clinical epidemiology 62: e1-34.

39. Rondón LJ, Groenestege WM, Rayssiguier Y, Mazur A (2008) Relationship between low magnesium status and TRPM6 expression in the kidney and large intestine. American journal of physiology. Regulatory, integrative and comparative physiology 294: R2001-R2007.

40. Mackay JD, Bladon PT (2010) Hypomagnesaemia due to proton-pump inhibitor therapy: a clinical case series. QJM 103: 387-395.

41. Furlanetto TW, Faulhaber GA (2011) Hypomagnesemia and proton pump inhibitors: below the tip of the iceberg. Archives of internal medicine 171: 13911392.

42. Liamis G, Rodenburg EM, Hofman A, Zietse R, Stricker BH, et al. (2013) Electrolyte disorders in community subjects: prevalence and risk factors. The American journal of medicine 126: 256-263.

43. Khan AM, Lubitz SA, Sullivan LM, Sun JX, Levy D, et al. (2013) Low serum magnesium and the development of atrial fibrillation in the community: the Framingham Heart Study. Circulation 127: 33-38.

44. Reffelmann T, DÃrr M, Ittermann T, Schwahn C, VÃlzke H, et al. (2010) Low serum magnesium concentrations predict increase in left ventricular mass over 5 years independently of common cardiovascular risk factors. Atherosclerosis 213: 563-569.

45. Famularo G, Gasbarrone L, Minisola G (2013) Hypomagnesemia and protonpump inhibitors. Expert Opinion on Drug Safety 12: 709-716.

46. Corleto VD, Festa S, Di Giulio E, Annibale B (2014) Proton pump inhibitor therapy and potential long-term harm. Current opinion in endocrinology, diabetes and obesity $21: 3-8$. 